\title{
Juvenile idiopathic arthritis (JIA) and early diagnosis of temporo-mandibular joint (TMJ) disorders
} MG Alpigiani*1, F Baldi ${ }^{1}$, A Calcagno ${ }^{1}$, P Salvati ${ }^{1}$, R Servetto ${ }^{2}$ and R Lorini ${ }^{1}$

Address: ${ }^{1}$ Institute G. Gaslini, Department of Pediatrics, University of Genova, Genova, Italy and 2Institute G. Gaslini, Division of Odontoiatric, University of Genova, Genova, Italy

* Corresponding author

from $15^{\text {th }}$ Paediatric Rheumatology European Society (PreS) Congress

London, UK. 14-17 September 2008

Published: 15 September 2008

Pediatric Rheumatology 2008, 6(SuppI I):P73 doi:I0.I I86/I546-0096-6-SI-P73

This abstract is available from: http://www.ped-rheum.com/content/6/SI/P73

(C) 2008 Alpigiani et al; licensee BioMed Central Ltd.

JIA is a chronic disease that can involve many different joints including TMJ. TMJ involvement leads to: chewing function alteration due to synovial membrane thickening, masticator muscle weakness and facial pain; craniofacial growth disturbance (micrognathia or facial asymmetry).

The aim of our study is to evaluate the TMJ in people affected by JIA for early diagnosis of TMJ arthritis.

Our series included 51 consecutive patients with JIA, 16 males and 35 females (age range 4 - 19 years), that were evaluated through a rheumatologic and dentist examination. Clinically suspected cases were studied with Magnetic Resonance Imaging (MRI). Our results showed that 19/51 patients (37\%) (age range 9 - 19 years) had TMJ alterations, $10 / 19(19,6 \%)$ concerning the left one while $7 / 19(13,7 \%)$ the right one. Only $2 / 19(4 \%)$ had a bilateral involvement.

TMJ arthritis can severely affect the growth of mandibular, it can be asymptomatic in the majority of cases but it is present in about $50 \%$ of children with JIA. MRI is useful to evidence the assessment of disease activity with precious informations about its evolution. Besides, this imaging technique allows to eliminate $\mathrm{X}$-rays exposure and, in according to its high definition, can show early joints alterations also in apparently asymptomatic children. In our patients, MRI was the gold standard for an early diagnosis showing that $37 \%$ of cases had TMJ involvement. 